\title{
Etiology associated with developing posthemispherectomy hydrocephalus after resection-disconnection procedures
}

\author{
Clinical article
}

\author{
Jennifer Phung, B.S., ${ }^{6}$ Paul Krogstad, M.D., ${ }^{2,3,6}$ and Gary W. Mathern, M.D. ${ }^{1,4-6}$ \\ ${ }^{1}$ Departments of Neurosurgery and Psychiatry \& Biobehavioral Sciences; ${ }^{2}$ Department of Pediatrics; \\ ${ }^{3}$ Department of Molecular and Medical Pharmacology; ${ }^{4}$ The Intellectual and Developmental Disabilities \\ Research Center; ${ }^{5}$ The Brain Research Institute, Mattel Children's Hospital; and ${ }^{6}$ David Geffen School of \\ Medicine, University of California, Los Angeles, California
}

Object. The authors sought to determine if clinical epilepsy variables, maximum daily temperature (Tmax), and blood and CSF findings were associated with the risk of developing hydrocephalus after first-time resectiondisconnection hemispherectomy.

Methods. Patients who underwent cerebral hemispherectomy in whom a standardized perioperative protocol was used, including the use of ventriculostomies $(n=79)$, were classified into those who developed and those who did not develop hydrocephalus requiring CSF shunts. The authors compared these 2 groups for clinical variables, Tmax, and blood and CSF studies through postoperative Day 12.

Results. In this cohort, $30 \%$ of the patients required CSF shunts, of which $8 \%$ developed late hydrocephalus up to 3 years posthemispherectomy. Multivariate analysis found that etiology was associated with developing posthemispherectomy hydrocephalus. Higher shunt rates were observed for patients with hemimegalencephaly $(40 \% ; n$ $=15)$ and a history of CNS infection $(100 \% ; n=4)$ compared with cortical dysplasia $(17 \% ; n=23)$ and Rasmussen encephalitis $(17 \% ; \mathrm{n}=12)$. In univariate analysis, other factors associated with developing hydrocephalus were elevated maximum daily temperatures, elevated white blood cell counts, decreased CSF protein, and increased CSF red blood cell counts.

Conclusions. The findings of the study indicate that etiology was the factor most strongly associated with developing posthemispherectomy hydrocephalus. These findings suggest that there are variable mechanisms for developing hydrocephalus after cerebral hemispherectomy depending on the procedure, and in resection-disconnection operations the mechanism may involve changes in CSF bulk flow that varies by histopathology.

(http://thejns.org/doi/abs/10.3171/2013.8.PEDS13212)

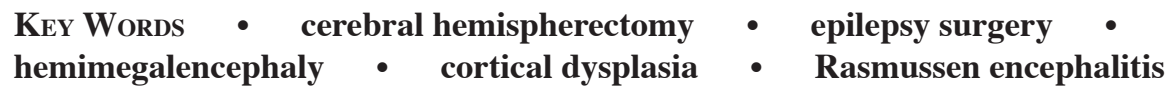

$\mathrm{H}$ YDROCEPHALUS is an unfortunate "side effect" of cerebral hemispherectomy; its reported incidence ranged from $9 \%$ to $81 \% .^{5-7,14,19,21}$ Based on concepts from developing de novo hydrocephalus, 3 possible causes of posthemispherectomy hydrocephalus may be the following: 1) blood and protein exuded from residual brain tissue could obstruct CSF absorption at the arachnoid granulations; 2) surgery could induce inflammatory processes that interfere with CSF absorption; and 3) disconnection of different brain areas from each another could have a deleterious effect on postoperative CSF flow dynamics. ${ }^{8,13,16,20}$ Previous studies, including

\footnotetext{
Abbreviations used in this paper: $\mathrm{RBC}=$ red blood cell; $\mathrm{Tmax}=$ maximum daily temperature; UCLA = University of California, Los Angeles; WBC = white blood cell.
}

a recent multicenter collaborative project involving different hemispherectomy techniques, have identified type of procedure and previous operations as factors associated with developing posthemispherectomy hydrocephalus. ${ }^{6,14,15}$ However, these reports were unable to clearly identify possible links with clinical variables that might suggest mechanisms for developing hydrocephalus after a particular hemispherectomy procedure.

The aim of the present study was to determine if clinical variables of epilepsy, such as etiology, and postsurgery variables, such as maximum daily temperature (Tmax), along with blood and CSF studies were associated with developing hydrocephalus after cerebral hemispherectomy, with the goal of identifying possible clinical mechanisms. Given prior findings, we concentrated on a single, firsttime resection-disconnection hemispherectomy operation 
using a standardized perioperative protocol including use of ventriculostomy catheters to control for prior surgeries and operative techniques. ${ }^{12,15}$ Based on clinical experience, we hypothesized that etiology would be the most important factor associated with developing posthemispherectomy hydrocephalus requiring CSF shunts when using our resection-disconnection procedure.

\section{Methods}

\section{Standard Protocol Approvals, Registrations, and Patient Consent}

This study was approved by the institutional review board at the University of California, Los Angeles (UCLA), and since enactment of HIPAA (Health Insurance Portability and Accountability Act), patients and families have signed research informed consent and authorization forms. This study was not a clinical trial, and it is not registered in any public registry.

\section{Study Population}

This was a retrospective review of consecutive cerebral hemispherectomy cases at UCLA's Pediatric Epilepsy Program from 2004 to 2012. Electronic medical records $(n=95)$ were searched for clinical information including pediatric ICU, nursing notes, and blood and CSF laboratory data. Cases were excluded from the study if the patients had undergone a previous hemispherectomy and were undergoing repeat procedures $(n=8)$ and if their first operation was lobar or multilobar resection and this was being converting to a cerebral hemispherectomy (n $=8$ ). The remaining 79 patients, whose procedures were first-time operations, constituted the study population.

All operations were performed by a single surgeon (G.W.M.), using a standardized cerebral hemispherectomy technique involving resection-disconnection and perioperative protocol pertaining to ventriculostomy and shunts. ${ }^{6,12}$ Ventriculostomy catheters were placed in the operating room prior to closure of the dura and were not changed after the hemispherectomy. Samples of CSF, beginning on the 2nd postoperative day, were obtained daily from the closed loop ventriculostomy system and sent for cell analysis, culture, and chemistry studies. Ventriculostomy catheters were removed if an overnight test clamp did not result in intracranial pressures greater than $18 \mathrm{~cm} \mathrm{H}_{2} \mathrm{O}$. In cases in which the test failed on 2 separate night trials, a ventriculoperitoneal shunt was placed. Test clamps were performed when, over the preceding 24 hours, CSF output was less than $75 \mathrm{ml}$ at $5 \mathrm{~cm}$ of $\mathrm{H}_{2} \mathrm{O}$ and CSF protein was less than $500 \mathrm{mg} / \mathrm{dl}$. Antibiotics were continued until 24 hours after external ventriculostomy drains were removed or ventriculoperitoneal shunt placed. Dexamethasone ( $1 \mathrm{mg} / \mathrm{kg}$ up to $10 \mathrm{mg}$ ) was given preoperatively and tapered over 4-5 days. The blood supply to the choroid plexus was not interrupted during the operation, and use of large volumes of hemostatic agents was discouraged when using our hemispherectomy technique. $^{15}$

Other components of the perioperative protocol were designed to reduce infections, as previously described. ${ }^{17}$
Antibiotic agents (cefazolin or, for allergic patients, clindamycin or vancomycin) were administered intravenously prior to skin incision and were continued postoperatively as noted above. In the operating room, Foley catheters and arterial and central lines (triple lumen; subclavian, jugular, or groin) were placed using sterile technique after induction of anesthesia. After surgery, blood samples were generally drawn daily and as necessary (usually for elevated temperatures) from the central line and sent for culture, routine counts, and chemistry. Foley catheters and most peripheral venous and arterial lines were discontinued within 48 hours of surgery. Central venous lines were removed at hospital discharge or changed to peripheral venous lines after 2 weeks. Blood, urine, and pulmonary samples were sent for bacterial culture in patients experiencing fevers (temperature $\geq 38.5^{\circ} \mathrm{C}$ ). The day of surgery was defined as Day 0.

\section{Clinical Data}

The presurgical clinical protocols and evaluation process, as well as methods describing collection of clinical epilepsy variables, postsurgery seizure outcome, and complications, have been previously detailed.,10 Seizure etiology categories, which were based on MRI and histopathological features, include the following: hemimegalencephaly in which 3 of 4 lobes were larger than expected; ${ }^{18}$ hemispheric or multilobar cortical dysplasia; perinatal cerebral ischemia/infarction (stroke); Rasmussen encephalitis; history of mostly unilateral CNS infection (3 cases of herpes simplex virus and 1 case of prior bacterial encephalitis); Sturge-Weber syndrome; and other (2 cases of extensive tumors [1 dysembryoplastic neuroepithelial tumor and 1 ganglioglioma]), history of cerebral trauma $(n=2)$, and deep thalamic damage and seizures after a prior ventricular endoscopic procedure $(n=1) .^{6}$

Additional information gathered for this study included maximum daily temperature (Tmax), blood white blood cell (WBC) count and differential, CSF laboratory results, and incidence and location of positive bacterial cultures for the first 12 days posthemispherectomy. ${ }^{17}$ As stated, a fever was defined as temperature of $38.5^{\circ} \mathrm{C}$ or higher, documented at least once on a given day. An elevated blood WBC count was defined as greater than 15,000 cells $/ \mu 1$.

\section{Study Design and Statistical Analysis}

Patients were retrospectively classified into those who developed and those who did not develop hydrocephalus requiring CSF shunts. These groups were compared for differences in clinical epilepsy variables, Tmax, and blood and CSF counts and chemistries; the statistical program StatView 5 (SAS Institute, Inc.) was used. ${ }^{3}$ Statistical tests included repeated-measures ANOVA and chi-square when appropriate. All tests were 2-tailed and the threshold for significance was set a priori at $\mathrm{p}<0.05$.

\section{Results}

\section{Cohort Description and Clinical Comparisons}

In this cerebral hemispherectomy cohort from 2004 
to $2012,24(30 \%)$ of 79 patients developed hydrocephalus requiring CSF shunts. All but 2 patients were successfully weaned from the perioperative ventricular catheter in the first 2 weeks after surgery; these 2 patients (8\%; both hemimegalencephaly cases) had developed late-onset hydrocephalus 1 and 3 years after surgery (Fig. 1). For comparison, the rate of CSF shunt placement in our patients from 1998 to the end of 2003 was $31 \%$ (20 of 64 patients) using the same operative technique. All except 3 patients had a single VP shunt without revision, and the other 3 have had 2,3, and 8 revisions, respectively, to date. One of these 3 patients with multiple shunt revisions developed a shunt infection, and the other cases have had proximal and distal obstructions. Comparison of clinical epilepsy variables except seizure etiology showed no differences in those who required shunts compared with those that did not (Table 1). By etiology, higher shunt rates were observed for those with hemimegalencephaly $(40 \% ; n=15)$ and a prior history of CNS infection $(100 \% ; n=4)$ compared with cortical dysplasia $(17 \%$; $n=23)$ and Rasmussen encephalitis $(17 \% ; n=12)$.

\section{Maximum Daily Temperature and Blood and CSF Studies}

In univariate analyses, differences were identified for Tmax as well as for blood and CSF studies in patients with and without shunts placed after cerebral hemispherectomy (Table 1; Fig. 2). For Tmax, repeated-measures testing over the first 12 days posthemispherectomy showed that those in whom CSF shunts were eventually required had higher temperatures (Fig. 2A, graph on left side). Inspection by individual days showed this was a general difference over the first 12 days and trended toward significance only on postsurgery Day 8 (Fig. 2A, graph on right side). For blood WBC counts, repeated-
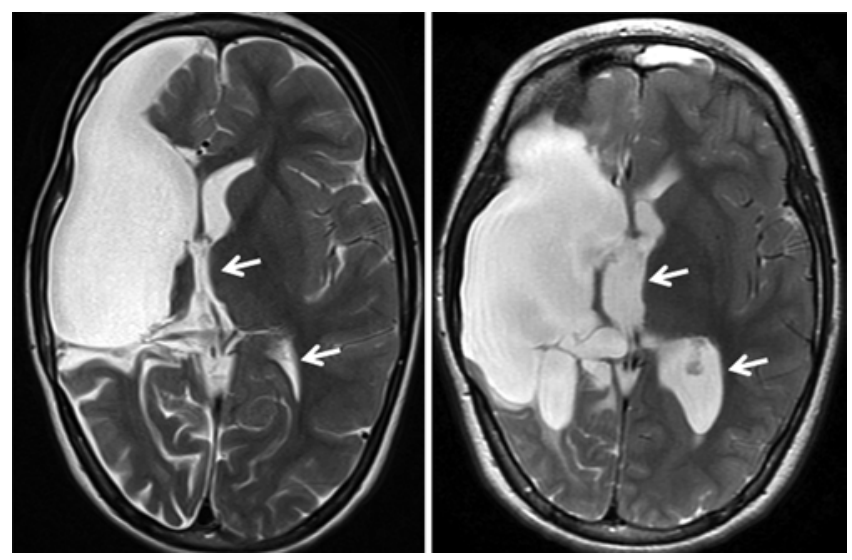

Fig. 1. Example of delayed hydrocephalus after cerebral hemispherectomy. This child underwent right hemispherectomy at age 6 years for Type I cortical dysplasia and has remained seizure free since surgery. ${ }^{4}$ Her MRI studies were stable for the first 2 years postsurgery (left). Beginning at the end of the 3rd year posthemispherectomy, the patient complained of intermittent headache and some visual problems when reading fine print. Repeat MRI 4 years after the hemispherectomy (right) showed enlargement of the third and left lateral ventricles (compare arrows in 2-year and 4-year studies). Her clinical symptoms subsided spontaneously and MRI has remained stable for another year. The patient's family declined CSF shunting because the clinical symptoms resolved and imaging status remained stable. measures ANOVA showed that patients receiving shunts had higher counts (Fig. 2B, graph on left side) that trended toward significance from postsurgery Day 10 to 12 . Results indicated that patients with shunts had lower CSF protein levels (Fig. 2C, graph on left side) that trended toward significance on postsurgery Days 7 and 9 (Fig. $2 \mathrm{C}$, graph on right side). For CSF red blood cell (RBC) counts, repeated-measures ANOVA showed that patients with shunts had higher counts (Fig. 2D, left graph) that were significant on postsurgery Day 6. In fact, for Days 6 and 7 posthemispherectomy, patients eventually requiring CSF shunts had higher CSF RBC counts than patients not needing shunts (ANOVA, $\mathrm{p}=0.0041$ ). For those 2 days posthemispherectomy, if the CSF RBC count was $100,000 / \mu l$ or greater, the sensitivity of requiring a shunt was $43 \%$ and specificity was $79 \%$ with an accuracy of $67 \%$ (Table 2).

\section{Multivariate Analysis}

To determine which variables were most strongly associated with requiring a shunt posthemispherectomy, we performed a logistical regression analysis in which etiology, Tmax, blood WBC, CSF protein, and CSF RBC counts were independent variables, and shunt versus noshunt was the dependent variable. We found that etiology $(\mathrm{p}=0.0001)$ was associated with developing posthemispherectomy hydrocephalus, whereas Tmax $(\mathrm{p}=0.775)$, blood WBC counts $(\mathrm{p}=0.107)$, CSF protein levels $(\mathrm{p}=$ 0.052), and CSF RBC counts $(\mathrm{p}=0.105)$ were not.

\section{Discussion}

In multivariate analysis of patients undergoing firsttime cerebral hemispherectomy that involved a resectiondisconnection technique, we observed that etiology was most strongly associated with developing postsurgery hydrocephalus requiring CSF shunts. Less strongly associated but significant in the univariate analysis in terms of developing hydrocephalus were maximum daily temperatures, elevated blood WBC counts, decreased CSF protein levels, and increased CSF RBC counts, especially on postsurgery Days 6 and 7 (Tables 1 and 2; Fig. 2).

Our findings and results from the literature help to define factors associated with the development of posthemispherectomy hydrocephalus, and these factors seem to depend on the type of operation that is performed. For example, in several studies surgeries that involve anatomical and hemidecortication operations that disconnect the arachnoid granulations and a history of another neurosurgical operation were factors linked with the development of hydrocephalus after cerebral hemispherectomy. ${ }^{6,14,15}$ Our study extends these findings by showing that, for a resection-disconnection procedure, etiology and, to a lesser extent, Tmax, and altered blood and CSF studies were associated with developing posthemispherectomy hydrocephalus. Prior studies may not have been able to identify seizure etiology as an important factor because they often grouped patients with hemimegalencephaly and cortical dysplasia into a single cortical maldevelopment group. Likewise, prior studies did not separate cases that involved a history of CNS infection from cases that 
TABLE 1: Clinical variables, Tmax, and blood and CSF studies stratified by patients who did and did not require CSF shunts for hydrocephalus after cerebral hemispherectomy*

\begin{tabular}{|c|c|c|c|}
\hline Variable & w/ Shunt $(n=24)$ & w/o Shunt $(n=55)$ & $p$ Value \\
\hline \multicolumn{4}{|l|}{ clinical variables } \\
\hline seizure onset (yrs) & $1.8 \pm 2.9$ & $2.0 \pm 3.7$ & 0.699 \\
\hline age at surgery (yrs) & $4.6 \pm 4.7$ & $5.2 \pm 4.4$ & 0.568 \\
\hline epilepsy duration (yrs) & $2.8 \pm 3.1$ & $3.2 \pm 2.9$ & 0.647 \\
\hline sex (female) & $54 \%(n=13)$ & $44 \%(n=24)$ & 0.388 \\
\hline side removed (It) & $58 \%(n=14)$ & $47 \%(n=26)$ & 0.366 \\
\hline etiology & & & 0.026 \\
\hline hemimegalencephaly $(n=15)$ & $40 \%(n=6)$ & $60 \%(n=9)$ & \\
\hline cortical dysplasia $(n=23)$ & $17 \%(n=4)$ & $83 \%(n=19)$ & \\
\hline ischemia/infarct $(n=18)$ & $33 \%(n=6)$ & $67 \%(n=12)$ & \\
\hline Rasmussen encephalitis $(n=12)$ & $17 \%(n=2)$ & $83 \%(n=10)$ & \\
\hline history of infection $(n=4)$ & $100 \%(n=4)$ & $0 \%(n=0)$ & \\
\hline Sturge-Weber $(n=3)$ & $33 \%(n=1)$ & $67 \%(n=2)$ & \\
\hline other $(n=4)$ & $25 \%(n=1)$ & $75 \%(n=3)$ & \\
\hline total complications $(n=17$ ) & $21 \%(n=5)$ & $21 \%(n=12)$ & 0.922 \\
\hline total infections $(n=14)$ & $21 \%(n=5)$ & $16 \%(n=9)$ & 0.368 \\
\hline CNS infections & $8 \%(n=2)$ & $4 \%(n=2)$ & 0.634 \\
\hline days ventriculostomy & $7.7 \pm 3.8$ & $7.4 \pm 3.0$ & 0.775 \\
\hline days in hospital & $15.7 \pm 7.4$ & $13.4 \pm 5.3$ & 0.137 \\
\hline seizure free at last follow-up & $83 \%(n=20)$ & $83 \%(n=45)$ & 0.990 \\
\hline follow-up duration (yrs) & $2.9 \pm 1.7$ & $2.2 \pm 1.6$ & 0.069 \\
\hline $\begin{array}{l}\text { Tmax in }{ }^{\circ} \mathrm{C} \text { (repeated-measures) } \\
\text { blood counts (repeated-measures) }\end{array}$ & \multicolumn{2}{|c|}{ blood counts (repeated-measures) } & 0.0029 \\
\hline WBC counts $\times 100$ & $13.2 \pm 4.6$ & $12.2 \pm 4.0$ & 0.016 \\
\hline$\%$ PMN & $59.7 \pm 14.6$ & $60.0 \pm 17.6$ & 0.983 \\
\hline \% lymphocytes & $27.2 \pm 12.6$ & $25.4 \pm 12.3$ & 0.286 \\
\hline$\%$ monocytes & $11.2 \pm 5.0$ & $10.9 \pm 3.7$ & 0.124 \\
\hline$\%$ eosinophil & $1.0 \pm 1.3$ & $1.3 \pm 1.7$ & 0.261 \\
\hline$\%$ basophils & $0.2 \pm 0.1$ & $0.2 \pm 0.2$ & 0.060 \\
\hline \multicolumn{4}{|l|}{ CSF (repeated-measures) } \\
\hline protein (mg/dl) & $540 \pm 390$ & $637 \pm 417$ & 0.033 \\
\hline WBC counts per $\mathrm{cm}^{3}$ & $833 \pm 1976$ & $699 \pm 2363$ & 0.593 \\
\hline $\mathrm{RBC}$ counts per $\mathrm{cm}^{3}$ & $98,194 \pm 167,842$ & $66,205 \pm 138,569$ & 0.049 \\
\hline $\mathrm{RBC} / \mathrm{WBC}$ ratio & $0.05 \pm 0.30$ & $0.03 \pm 0.09$ & 0.254 \\
\hline$\%$ PMN & $74.9 \pm 22.4$ & $75.0 \pm 24.9$ & 0.990 \\
\hline$\%$ bands & $1.3 \pm 0.6$ & $1.3 \pm 0.5$ & 0.827 \\
\hline$\%$ lymphocytes & $11.6 \pm 15.4$ & $12.1 \pm 15.5$ & 0.808 \\
\hline$\%$ monocytes & $13.2 \pm 12.6$ & $14.3 \pm 15.7$ & 0.526 \\
\hline \% eosinophil & $5.1 \pm 8.8$ & $1.7 \pm 1.4$ & 0.052 \\
\hline
\end{tabular}

* Mean values are presented \pm SD. Significant values $(p<0.05)$ are indicated in boldface. PMN = polymorphonuclear leukocytes.

involved other atrophic etiologies such as perinatal infarcts. Based on our findings, we recommend that future studies have large enough sample sizes to allow classification of patients into appropriate seizure etiology categories according to different procedures, so as to identify clinical factors associated with the development of posthemispherectomy hydrocephalus.

Our findings also begin to suggest that pathophysi- ological mechanisms leading posthemispherectomy hydrocephalus will vary by procedure and etiology. Larger resections that involve obliteration of half of the arachnoid granulations could lead to hydrocephalus as a result of reduced CSF absorption. In the case of resection-disconnection procedures, our data support the concept that alternate mechanisms may contribute to developing posthemispherectomy hydrocephalus involving differences 
Cerebrospinal fluid shunts after hemispherectomy
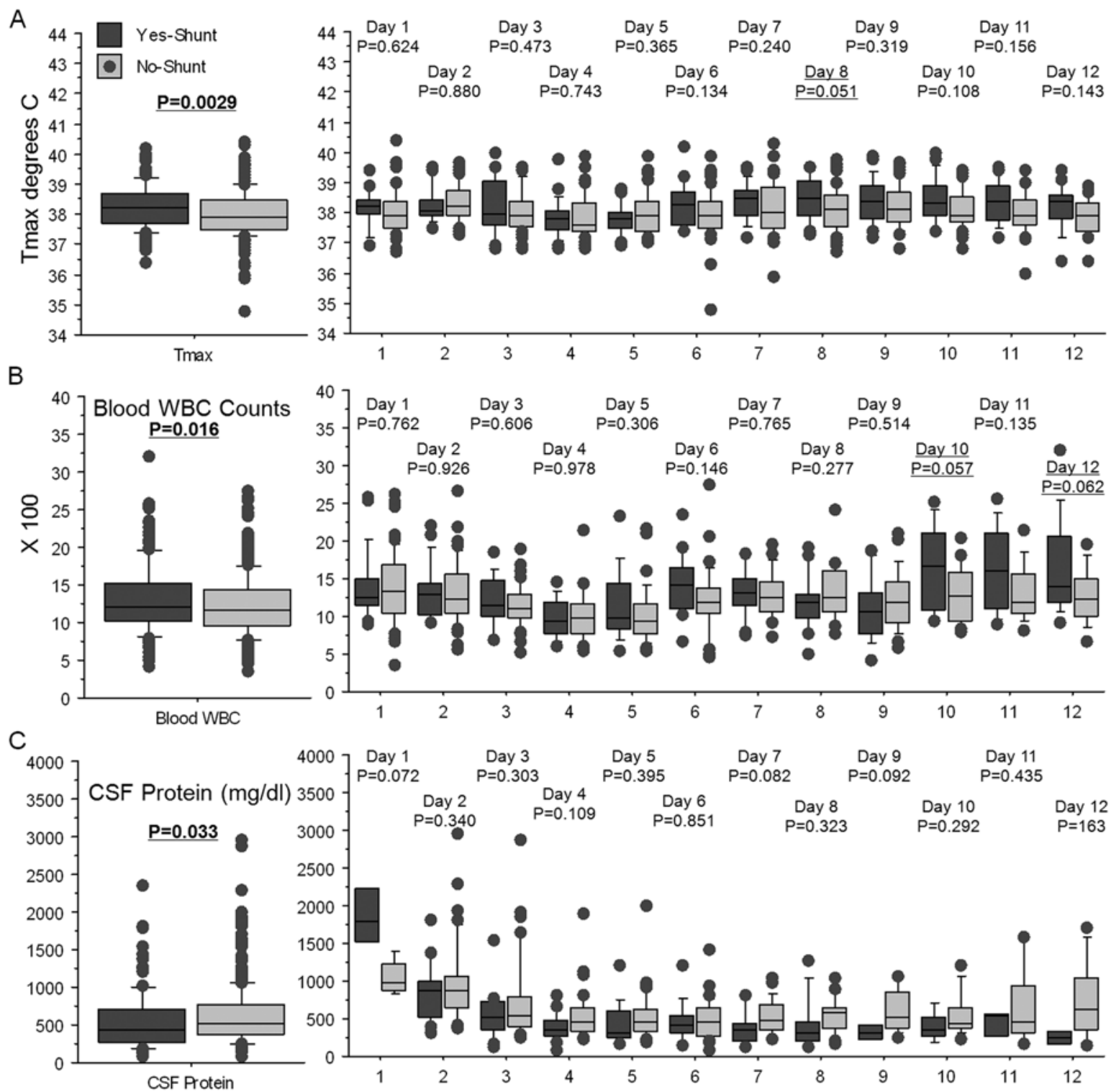

D

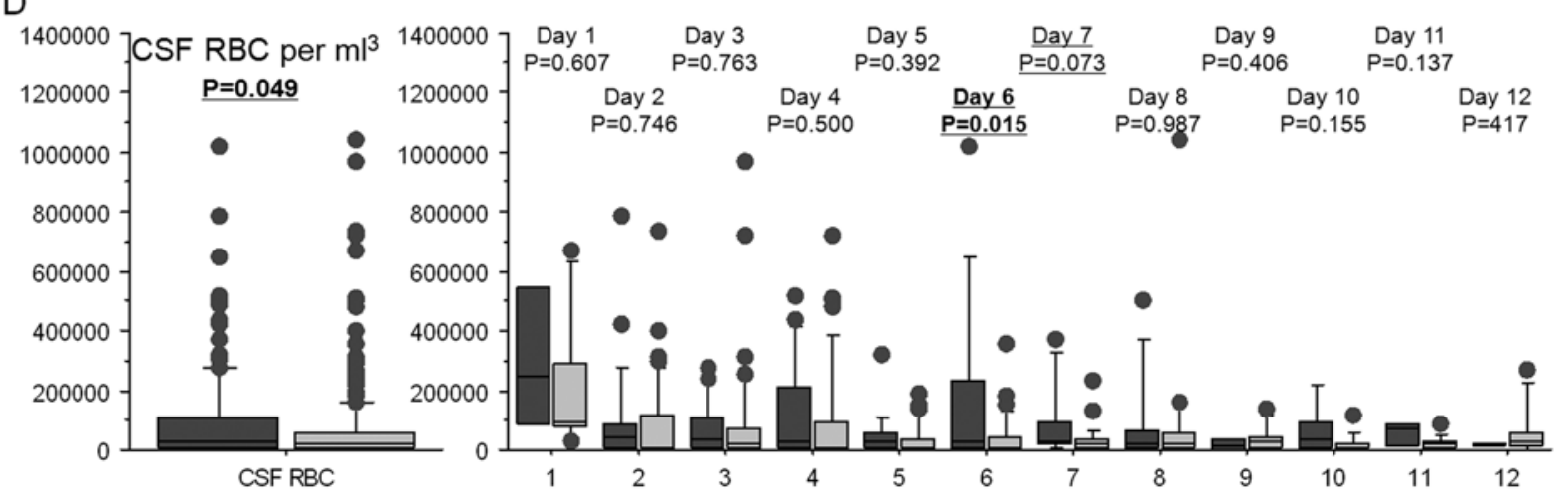

FIG. 2. Box plots displaying Tmax (A), blood WBC counts (B), CSF protein levels (C), and CSF RBC counts (D) for patients with (dark gray bars) and without (light gray bars) shunts. Results of repeated-measures ANOVA are shown for all days presented (left) and individual days from 1 to 12 (right). Probability values are shown above each plot with significant values $(p<0.05)$ indicated by boldface and trends $(p<0.07)$ indicated by underlining. 
TABLE 2: Sensitivity, specificity, and accuracy of CSF RBC counts of 100,000/pl or greater on posthemispherectomy Days 6 and 7 in predicting the need for CSF shunts*

\begin{tabular}{cccc}
\hline & \multicolumn{3}{c}{ No. of Patients } \\
\cline { 2 - 4 } CSF RBC & w/ Shunt & w/o Shunt & Total \\
\hline$>100,000 / \mu 1$ & 7 & 7 & 14 \\
$<100,000 / \mu 1$ & 9 & 26 & 35 \\
total & 16 & 33 & 49 \\
\hline
\end{tabular}

* Data based on on 54 patients who still had ventriculostomy catheters in place. Cerebrospinal fluid was not drawn in every patient every day.

in etiology and histopathology. For example, we suggest that after resection-disconnection procedures, the residual brain tissue and subarachnoid space appear to be less able to absorb and diffuse CSF to the arachnoid granulations in cases of hemimegalencephaly and prior infections compared with cases of Rasmussen encephalitis. Such findings support the concept that CSF flow within the brain is probably altered related to the severe cortical and white matter disorganization in hemimegalencephaly cases rather than changes in brain compliance or due to blood and tissue debris since CD cases had lower rates of needing shunts despite similar ages at surgery, ventricular size, and expected blood loss during surgery., ${ }^{1,2,11}$ Alternately, differences in etiology may contribute to the ability of the arachnoid granulations to remain open after resection-disconnection hemispherectomy. While acute changes in CSF osmolarity reflected by increased CSF RBC counts may also contribute to developing hydrocephalus, our data suggest that this is a minor association. Moreover, in our cohort we found no clear associations with changes in CSF WBC counts to indicate involvement of brain inflammation as a likely etiology of hydrocephalus. These notions will require confirmation in future hypothesis-driven investigations, but the current study provides clinical observations that support possible pathophysiological mechanisms for development of posthemispherectomy hydrocephalus that are strongly associated with procedure and etiology.

The reader should be aware of the inherent limitations of this study. This was a retrospective study, and blood laboratory studies were not performed daily for every patient as part of the perioperative protocol. After the first few days, blood samples were sent for testing, as clinically indicated (usually for elevated temperatures), and we estimate that blood work was obtained from $90 \%$ of patients in the first 3 days postsurgery, and this rate declined to about 50\% from Day 4 onward. Future prospective studies should include daily blood laboratory studies as part of the research design. Daily CSF studies from Day 2 onward were a part of the clinical protocol and are therefore more reliable. Furthermore, our results identified associations between clinical variables and laboratory studies in terms of developing hydrocephalus after hemispherectomy. We should emphasize that our findings reflect associations between etiology and clinical variables, not causality. In the case of etiology, for example, there are strongly linked covariables, such as age at seizure onset and surgery, blood loss during surgery, and length of the operation, that could be linked with the development of posthemispherectomy hydrocephalus not tested for in our research design. ${ }^{6,12}$ Finally, our results are most pertinent for pediatric patients undergoing cerebral hemispherectomy for epilepsy surgery. It is unclear if similar results would be found for other types of pediatric and adult neurosurgical nonepilepsy procedures.

\section{Conclusions}

In multivariate analysis, this study found that etiology was the clinical factor most closely associated with developing posthemispherectomy hydrocephalus when our resection-disconnection procedure was performed. Weaker associations with hydrocephalus found in univariate analysis included increased maximum daily temperature (Tmax), increased blood WBC and CSF RBC counts, and decreased CSF protein levels. These findings support the hypothesis that the most likely mechanism for developing hydrocephalus after our cerebral hemispherectomy procedure involved changes in CSF flow dynamics that varied by etiology. Our findings are helpful for practicing neurosurgeons in predicting which patients are at greatest risk for developing hydrocephalus after cerebral hemispherectomy involving resection-disconnection procedures.

\section{Acknowledgments}

The authors acknowledge the following individuals for their contribution to the UCLA pediatric epilepsy surgery program during the time of this study: W. Donald Shield, M.D., Joyce Wu, M.D., Joyce Matsumoto, M.D., Jason Lerner, M.D., Shaun Husain, M.D., Christopher C. Giza, M.D., and Sue Yudovin, R.N., N.P.

\section{Disclosure}

The study was supported in part by an NIH grant (no. R01 NS38992) to Dr. Mathern and the Short Term Training Program at David Geffen School of Medicine at UCLA to Ms. Phung.

The authors report no financial conflict of interest concerning the materials or methods used in this study or the findings specified in this paper.

Author contributions to the study and manuscript preparation include the following. Conception and design: Mathern. Acquisition of data: Phung. Analysis and interpretation of data: Mathern. Drafting the article: all authors. Critically revising the article: all authors. Reviewed submitted version of manuscript: all authors. Approved the final version of the manuscript on behalf of all authors: Mathern. Statistical analysis: Mathern.

\section{References}

1. Akhtari M, Mandelkern M, Bui D, Salamon N, Vinters HV, Mathern GW: Variable anisotropic brain electrical conductivities in epileptogenic foci. Brain Topogr 23:292-300, 2010

2. Akhtari M, Salamon N, Duncan R, Fried I, Mathern GW: Electrical conductivities of the freshly excised cerebral cortex in epilepsy surgery patients; correlation with pathology, seizure duration, and diffusion tensor imaging. Brain Topogr 18:281-290, 2006

3. Baca CB, Vickrey BG, Vassar S, Hauptman JS, Dadour A, Oh T, et al: Time to pediatric epilepsy surgery is related to disease severity and nonclinical factors. Neurology 80:1231-1239, 2013 


\section{Cerebrospinal fluid shunts after hemispherectomy}

4. Blümcke I, Thom M, Aronica E, Armstrong DD, Vinters HV, Palmini A, et al: The clinicopathologic spectrum of focal cortical dysplasias: a consensus classification proposed by an ad hoc Task Force of the ILAE Diagnostic Methods Commission. Epilepsia 52:158-174, 2011

5. Carson BS, Javedan SP, Freeman JM, Vining EP, Zuckerberg AL, Lauer JA, et al: Hemispherectomy: a hemidecortication approach and review of 52 cases. J Neurosurg 84:903-911, 1996

6. Cook SW, Nguyen ST, Hu B, Yudovin S, Shields WD, Vinters $\mathrm{HV}$, et al: Cerebral hemispherectomy in pediatric patients with epilepsy: comparison of three techniques by pathological substrate in 115 patients. J Neurosurg 100 (2 Suppl Pediatrics): 125-141, 2004

7. Davies KG, Maxwell RE, French LA: Hemispherectomy for intractable seizures: long-term results in 17 patients followed for up to 38 years. J Neurosurg 78:733-740, 1993

8. Di Rocco C, Iannelli A: Hemimegalencephaly and intractable epilepsy: complications of hemispherectomy and their correlations with the surgical technique. A report on 15 cases. Pediatr Neurosurg 33:198-207, 2000

9. Hauptman JS, Pedram K, Sison CA, Sankar R, Salamon N, Vinters HV, et al: Pediatric epilepsy surgery: long-term 5-year seizure remission and medication use. Neurosurgery 71:985993, 2012

10. Hemb M, Velasco TR, Parnes MS, Wu JY, Lerner JT, Matsumoto $\mathrm{JH}$, et al: Improved outcomes in pediatric epilepsy surgery: the UCLA experience, 1986-2008. Neurology 74:17681775,2010

11. Huang SY, Wolahan SM, Mathern GW, Chute DJ, Akhtari M, Nguyen ST, et al: Improving MRI differentiation of gray and white matter in epileptogenic lesions based on nonlinear feedback. Magn Reson Med 56:776-786, 2006

12. Jonas R, Nguyen S, Hu B, Asarnow RF, LoPresti C, Curtiss S, et al: Cerebral hemispherectomy: hospital course, seizure, developmental, language, and motor outcomes. Neurology 62: 1712-1721, 2004

13. Krishnamurthy S, Li J, Schultz L, McAllister JP II: Intraven- tricular infusion of hyperosmolar dextran induces hydrocephalus: a novel animal model of hydrocephalus. Cerebrospinal Fluid Res 6:16, 2009

14. Kwan A, Ng WH, Otsubo H, Ochi A, Snead OC III, Tamber MS, et al: Hemispherectomy for the control of intractable epilepsy in childhood: comparison of 2 surgical techniques in a single institution. Neurosurgery 67 (2 Suppl Operative):429-436, 2010

15. Lew SM, Matthews AE, Hartman AL, Haranhalli N: Posthemispherectomy hydrocephalus: results of a comprehensive, multiinstitutional review. Epilepsia 54:383-389, 2013

16. McComb JG: Recent research into the nature of cerebrospinal fluid formation and absorption. J Neurosurg 59:369-383, 1983

17. Phung J, Mathern GW, Krogstad P: Timing and predictors of fever and infection after craniotomy for epilepsy in children. Pediatr Infect Dis J 32:450-459, 2013

18. Salamon N, Andres M, Chute DJ, Nguyen ST, Chang JW, Huynh MN, et al: Contralateral hemimicrencephaly and clinical-pathological correlations in children with hemimegalencephaly. Brain 129:352-365, 2006

19. Schramm J, Kuczaty S, Sassen R, Elger CE, von Lehe M: Pediatric functional hemispherectomy: outcome in 92 patients. Acta Neurochir (Wien) 154:2017-2028, 2012

20. Welch K, Friedman V: The cerebrospinal fluid valves. Brain 83:454-469, 1960

21. Wilson PJ: Cerebral hemispherectomy for infantile hemiplegia. A report of 50 cases. Brain 93:147-180, 1970

Manuscript submitted April 30, 2013.

Accepted August 5, 2013.

Please include this information when citing this paper: published online September 6, 2013; DOI: 10.3171/2013.8.PEDS13212.

Address correspondence to: Gary W. Mathern, M.D., UCLA Neurosurgery, 710 Westwood Plaza, Rm. 2123, Los Angeles, CA 90095.email: gmathern@ucla.edu. 